\title{
The hunt for ancient novae
}

\author{
Linda Schmidtobreick* \\ ESO, Chile \\ E-mail: Ischmidt @eso org
}

\section{Claus Tappert}

Universidad de Valparaíso, Chile

\section{Michael Shara}

AMNH, USA

\section{Simone Scaringi}

University of Canterbury, $N Z$

\section{Amelia Bayo \\ Universidad de Valparaíso, Chile}

\section{Nikolaus Vogt}

Universidad de Valparaíso, Chile

\section{Alessandro Ederoclite \\ CEFCA, Spain}

\begin{abstract}
A nova eruption in a cataclysmic variable (CV) is a thermonuclear explosion on the surface of the white-dwarf primary once it has accreted a critical mass from its late-type companion. Between these eruptions, the binary is supposed to appear as a 'normal' CV, although for the first tens or hundreds of years, the white dwarf is still heated up which might influence the mass-transfer rate of the binary. To compare the nova-populations with the general CV-one, a large number of old novae are needed.

We have conducted a program to recover old novae and study the binary within. We here present the results of this program as well as a new deep wide-field $\mathrm{H} \alpha+[\mathrm{NII}]$ survey of cataclysmic variables to search for remnant nova shells around these objects. Such shells have been found around some cataclysmic variables that were hitherto not known as novae. They provide the unambiguous evidence that the system has experienced a nova eruption in the past and thus point to the oldest novae observable.
\end{abstract}

The Golden Age of Cataclysmic Variables and Related Objects IV

11-16 September, 2017

Palermo, Italy

${ }^{*}$ Speaker. 


\section{Introduction}

A nova eruption in a cataclysmic variable (CV) is a thermonuclear explosion on the surface of the white-dwarf primary once it has accreted a critical mass from its late-type companion. During the nova eruption, material is ejected into the interstellar medium, forming an expanding shell around the $\mathrm{CV}$ which can be observed once its angular size is sufficiently large to be resolvable from the inner binary (see e.g. [3]). In-between nova eruptions the binary is supposed to appear as a "normal" CV, i.e. its behaviour is dominated by its current mass-transfer rate and the magnetic field strength of the white dwarf [29]. However, the scenario of Shara et al. [16] predicts that the nova eruption affects the mass-transfer rate $\dot{M}$ on the long term: An initial phase of enhanced $\dot{M}$ is due to the irradiation of the secondary star by the eruption-heated white dwarf [4]. As the white dwarf cools, this irradiation is supposed to diminish resulting in a decline of $\dot{M}$ over the next centuries by several orders of magnitude, possibly even to the point that $\dot{M} \sim 0$ due to the secondary star losing contact to its Roche lobe, a scenario that has been termed "hibernation" [5].

There are several supports for the idea of such a cyclic nova - dwarf nova evolution as was originally proposed by Vogt [28]:

- The discovery of ancient nova shells around two low-mass transfer systems, i.e. Z Cam [17] and AT Cnc [18] as well as the discovery of a dwarf nova within the nova shell Te 11 [6], show without doubt, that dwarf novae have had nova eruptions in the past.

- The old nova GK Per shows typical dwarf-nova outbursts with amplitudes of a couple of magnitudes at intervals of several hundreds of days [10],[20]

- The existence of ER UMa stars and in particular of BKLyn, a nova-like star which since about 12 years has started to show dwarf-nova outbursts. Patterson et al. [9] interpret this behaviour by identifying BKLyn with a probable nova on 101 December 30 which took almost 2000 years to evolve from a nova-like to a dwarf-nova state.

- Three recently re-discovered old novae [13],[21] show optical spectra that are more representative of dwarf novae than of nova-like stars, and at least one of them, V728 Sco, also shows evidence for dwarf-nova like outbursts [22]

- The classical nova V1213 Cen has shown dwarf-nova outbursts in the six years before its eruption in 2009. It is now 2 mag brighter than as a pre-nova and shows a stable nova-like lightcurve [7]

However, all these systems could be individual cases indicating that dwarf novae also show nova eruptions without the need of a cyclic evolution. To conclude on this, a careful population study of novae, comparing high-mass transfer systems to low-mass transfer systems, is needed. We have started a project to establish a sample of ancient novae that can be used for such a population study and present the various attempts and their results in the following sections.

\section{The search for old novae}

To study the nature of the binary system decades after the nova eruption, we started a project in 2010 to establish the known population of old novae. The initial target list was selected using 
the catalogue by Downes et al. [2] including all classical novae that had an eruption before 1980, to have sufficient time passed for the underlying binary to become spectroscopically accessible. We took note of the fact that from the roughly 200 systems known, only 28 had a reliable orbital period and in fact a total of 141 post-novae lacked an identified candidate or a spectroscopic confirmation of the binary candidate.

We thus planned the project in three phases:

1. Photometric identification of nova candidates: We use two-colour diagrams to separate the CV from the bulk of stars in the field, for details on the method, see Tappert et al. [21].

2. Spectroscopic confirmation of the CV: Identified candidates are confirmed as CVs by optical spectroscopy [21],[24],[25],[26]

3. Determination of the orbital period: Time resolved photometry or spectroscopy is performed on the brightest confirmed candidates with the aim to measure the orbital period [22],[23]

For the most recent results, see the proceedings by Ederoclite et al. in this conference. Here is a summary of the main results of this project: We increased the knowledge on the CV population that experienced a nova eruption in the past and can compare it with the general CV population. Most of the old novae are situated above the period gap. The overabundance of systems between 3 and 4 hours is present as in the 'normal' CVs. While we do find a couple of systems that show indications of low mass transfer, the great majority of the old novae are high mass-transfer systems. We conclude that any cyclic evolution, if present, has to take place on time-scales longer than $\approx 150$ years, the typical oldest age of observed classical novae.

\section{The search for ancient novae}

To find the novae that are older than these $\approx 150$ years, a different approach has to be taken. Shara et al. have shown that ancient novae of 2000 years age, can be identified via their remnant shells [17],[18]. We thus use this idea to find ancient novae among the population of known CVs. Deep, wide-field images in $\mathrm{H} \alpha+\mathrm{N}[\mathrm{II}]$ have been taken of $15 \mathrm{CVs}$ of low mass-transfer rate with periods between 3 and 4 hours but no detection of a remnant shell has been made. Including our detection limit, the percentage of novae that show shells and the number of observed systems we estimated a lower limit of 13000 years for the recurrence time of these systems. This is well in agreement to what is expected for such CVs with an average mass-transfer rate of about $5 \times 10^{-10} \mathrm{M}_{\odot} / \mathrm{yr}$ : The white dwarf needs to accrete for at least 20000 years to reach the critical mass for the nova explosion [14]. We also observed 20 novalike stars down to a limit of $10^{-17} \mathrm{erg} \mathrm{cm}^{-2} \mathrm{~s}^{-1} \operatorname{arcsec}^{-2}$ but without detection [15]. Increasing the statistical accuracy by using a tight $95 \%$ confidence interval, we find a lower limit of the recurrence time of 9000 years. This is in contradiction with the recurrence time of 4000 years, expected for an average high mass-transfer system with $5 \times 10^{-9} \mathrm{M}_{\odot} / \mathrm{yr}$.

We compare our finding to those of other searches for nova shells: Sahman et al. searched 24 novalike stars with shallow exposures and found a shell around V1315 Aql, the result of a nova eruption approximately 120 years ago [12]. As such, the nova is one that could also have been spotted in eruption and is not really of ancient type. In fact, their observations with shallow 
exposures were targeted at discovering "recent" nova eruptions of about up to an age of about 220 yr. In a recent analysis, however, they discuss a possibly higher age for this nova remnant [11]. Pagnotta and Zurek find no detection of nova shells around asynchronous polars [8]. Shara et al. found a possible dwarf nova or intermediate polar in the shell of the nova 1437 A.D. [19].

The question arises why there are so few detection of nova shells. We acknowledge that there are many uncertainties, such as the average life span of a shell. While we know that 2000 year old shells exist, they might be the exception rather than the rule. Downes et al. analysed the evolution of shells decades after the eruption and were able to fit the decline by a straight line [1]. Using this fit and extrapolating the line, nova shells should become too faint to be easily detected after about 250 years which is in clear contradiction to the discoveries of Shara et al. Also, some of Downes' data might suggest that on longer time-scales, a plateau is reached when the nova shell does not become fainter anymore. This can be understood assuming that a large part of the shell emission at later stages arises from the interaction of the ejected material with the interstellar medium rather than from photoionisation. Clearly, an investigation on the general behaviour of old shells is needed. Such a project is presented by Tappert et al. in this conference [27].

If the low detection rate indeed indicates a deficiency of nova eruptions, then this deficiency is clearly on the side of the high mass-transfer systems. Of the 25 dwarf novae looked at, 4 shells have been found while among the 43 novalike stars, only one 'recent' shell has been found. We would like to point out that dwarf novae due to their lower mass-transfer rate should have on average longer recurrence times than novalike stars, and thus novalikes should be the ones more likely to show a shell. A tentative interpretation could be that there is a low mass-transfer phase in between the post-nova and pre-nova novalike phases and the majority of the novalikes that we observe and that were not observed as a nova in the last 200 years, belong to the pre-nova phase rather than the post-nova phase and as such had their last eruption many thousand years ago. This would support the theory of a cyclic evolution of the CVs in between nova eruptions.

However, there still remain many uncertainties and despite all the efforts undertaken to improve the numbers of these very old novae, we are still dealing with very small number statistics, so these conclusions have to be taken carefully.

\section{References}

[1] Downes, R. A., Duerbeck, H. W., Delahodde, C. E., 2001, Journal of Astronomical Data 7

[2] Downes, R. A., Webbink, R. F., Shara, M. M., Ritter, H., Kolb, U., Duerbeck, H. W., 2005, VizieR Online Data Catalog 5123

[3] Gill, C. D. and O’Brien, T. J., 1998, MNRAS 300, 221

[4] Kovetz, A., Prialnik, D., Shara, M. M., 1988, ApJ 325, 828

[5] Livio, M., Shara, M. M., 1987, ApJ 319, 819

[6] Miszalski, B., Woudt, P. A., Littlefair, S. P., et al., 2016, MNRAS 456, 633

[7] Mroz, P., Udalski, A., Pietrukowicz, P., et al., 2016, Nature 537, 649

[8] Pagnotta, A., Zurek, D., 2016, MNRAS 458, 1833

[9] Patterson, J., Uthas, H., Kemp, J., et al., 2013, MNRAS 434, 1902 
[10] Sabbadin, F., Bianchini, A., 1983, A\&AS 54, 393

[11] Sahman, D. I., Dhillon, V. S., Littlefair, S. P., Hallinan, G., 2018, MNRAS 477, 4483

[12] Sahman, D. I., Dhillon, V. S., Knigge, C., Marsh, T. R., 2015, MNRAS 451, 2863

[13] Schmidtobreick, L., Tappert, C., Bianchini, A., Mennickent, R. E., 2005, A\&A 432, 199

[14] Schmidtobreick, L., Shara, M., Tappert, C., Bayo, A., Ederoclite, A., 2015, MNRAS 449, 2215

[15] Schmidtobreick, L., Shara, M., Tappert, C., Scaringi, S., Bayo, A, Ederoclite, A., 2018, submitted to MNRAS

[16] Shara, M. M., Livio, M., Moffat,A. F. J., Orio, M., 1986, ApJ 311, 163

[17] Shara, M. M., Martin, C. D., Seibert, M., et al., 2007, Nature 446, 159

[18] Shara, M. M., Mizusawa, T., Wehinger, P., et al., 2012, ApJ 758, 121

[19] Shara, M. M., Iłkiewicz, K., Mikołajewska, J., 2017, Nature 548, 558

[20] Šimon, V., 2000, A\&A 354, 103

[21] Tappert, C., Ederoclite, A., Mennickent, R. E., Schmidtobreick, L., Vogt, N., 2012, MRAS 423, 2476

[22] Tappert, C., Vogt, N., Schmidtobreick, L., Ederoclite, A., Vanderbeke, J., 2013a, MNRAS 431, 92

[23] Tappert, C., Schmidtobreick, L., Vogt, N., Ederoclite, A., 2013b, MNRAS 436, 2412

[24] Tappert, C., Vogt, N., Della Valle, M., Schmidtobreick, L., Ederoclite, A., 2014, MNRAS 442, 565

[25] Tappert, C., Vogt, N., Schmidtobreick, L., Ederoclite, A., 2015, MNRAS 450, 943

[26] Tappert, C., Barria, D., Fuentes Morales, I., Vogt, N., Ederoclite, A., Schmidtobreick, L., 2016, MNRAS 462, 1371

[27] Tappert, C., Fuentes Morales, I., Puebla, E., Ederoclite, A., Schmidtobreick, L., Vogt, N., 2017, ArXiv e-prints 1702.02415

[28] Vogt, N., 1982, Mitteilungen der Astronomischen Gesellschaft Hamburg 57, 79

[29] Vogt, N., 1989, Classical Novae, ed. Bode \& Evans, p. 225 